\title{
Geometrical Theory of Diffraction Formulation for On-Body Propagation
}

\author{
Kammersgaard, Nikolaj Peter Brunvoll; Kvist, Søren H.; Thaysen, Jesper; Jakobsen, Kaj Bjarne
}

Published in:

IEEE Transactions on Antennas and Propagation

Link to article, DOI:

10.1109/TAP.2018.2882596

Publication date:

2019

Document Version

Peer reviewed version

Link back to DTU Orbit

Citation (APA):

Kammersgaard, N. P. B., Kvist, S. H., Thaysen, J., \& Jakobsen, K. B. (2019). Geometrical Theory of Diffraction Formulation for On-Body Propagation. IEEE Transactions on Antennas and Propagation, 67(2), 1143-1152. https://doi.org/10.1109/TAP.2018.2882596

\section{General rights}

Copyright and moral rights for the publications made accessible in the public portal are retained by the authors and/or other copyright owners and it is a condition of accessing publications that users recognise and abide by the legal requirements associated with these rights.

- Users may download and print one copy of any publication from the public portal for the purpose of private study or research.

- You may not further distribute the material or use it for any profit-making activity or commercial gain

- You may freely distribute the URL identifying the publication in the public portal 


\title{
Geometrical Theory of Diffraction Formulation for On-Body Propagation
}

\author{
Nikolaj P. B. Kammersgaard, Søren H. Kvist, Jesper Thaysen, and Kaj B. Jakobsen
}

\begin{abstract}
A Geometrical Theory of Diffraction model for onbody propagation is developed in the article. The exact solution to the canonical problem of a plane wave incident on an infinitely long cylinder, with arbitrary constitutive parameters, is found. The same is done for a magnetic and an electric infinitesimal dipole source of any orientation, located on the surface of the cylinder. The exact solutions are transformed with the Watson transformation to yield asymptotic expressions that are valid in the deep shadow region. These asymptotic expressions are validated by comparison to the numerically evaluated exact solution. It is found that the expressions are valid as long as the object is opaque, with a geometry down to the size of $\frac{\kappa}{\kappa^{2}+\tau^{2}}>\lambda_{0} / 2$, and the rays not too torsional $\tau / \kappa<2$, where $\kappa$ and $\tau$ are the curvature and torsion of the local geometry, respectively. The asymptotic expressions are found to approximate the exact solution significantly better than the asymptotic expression of an equivalent perfect electric conductor geometry. The same is the case for the impedance boundary condition asymptotic approximation for low dielectric constant materials. Finally, the asymptotic expressions are generalized so they can be applied to any convex geometry of the human body or an opaque lossy dielectric of electrically large size.
\end{abstract}

Index Terms-Geometrical Theory of Diffraction, asymptotic approximation, on-body communication, WBAN, lossy dielectric cylinder.

\section{INTRODUCTION}

$\mathbf{O}$ N-BODY antennas and propagation have received enormous attention in recent years. It has been a major research area, but has recently also resulted in many commercial uses. Within the past two years, for example, more than 10 sets of so-called 'truly' wireless headsets have been launched. The 'truly' wireless term refers to the fact that these headsets have independent left and right devices with no wire in-between. These devices are dependent on the following three kinds of connections; on-body communication between the ears or Earto-Ear (E2E) communication, on-body communication to for example a phone in the pocket or Pocket-to-Ear (P2E) communication, and off-body communication with any kind of music streaming device not placed on the body. Exactly the same user cases are present for modern BlueTooth ${ }^{\circledR}$ connected Hearing Instruments (HIs). For the HIs, the E2E communication is important in order to improve the acoustic performance. The E2E connection allows the two HIs' microphones on the left and right ear, respectively, to work together in a similar manner as an antenna array.

N. P. B. Kammersgaard and K. B. Jakobsen are with the Electromagnetic Systems Group, Department of Electrical Engineering, Technical University of Denmark, Kgs. Lyngby, 2800 Denmark (e-mail: npivka@elektro.dtu.dk).

N. P. B. Kammersgaard, S. H. Kvist and J. Thaysen are with GN Hearing A/S, Ballerup, 2750 Denmark.

Manuscript submitted January, 2018.
The exact understanding and modeling of the propagation of the fields on the surface of the body is crucial in order to design on-body wireless devices. The use of creeping wave theory has proven to be a good approximation in many cases. Primarily Geometrical Theory of Diffraction (GTD) or Uniform Geometrical Theory of Diffraction (UTD) have been applied to the problem of on-body propagation. Examples of the use of GTD or UTD can be found in [1]-[3]. In most cases the body is assumed to be a Perfect Electric Conductor (PEC) [3]-[6]. Others only investigate torsion free cases [1], [7], [8]. The validity of the PEC approximation as well as influence of torsion was briefly investigated in [9], [10].

A GTD/UTD formulation for the Impedance Boundary Condition (IBC) was done in [11], [12] and for dielectric coated cylinders in [13], [14]. These approximations would be more appropriate to use for on-body propagation than the PEC assumption. The IBC approximation is based on an assumption only valid for a material with high conductivity, however. This assumption is not fulfilled for human tissue at $2.45 \mathrm{GHz}$. The dielectric coated cylinders has a PEC core which is not a correct assumption for the human body either. Therefore, it is relevant to find a formulation valid for human tissue. However, the approaches used in [11] [14] are useful.

The purpose of this work is to provide a GTD formulation for the on-body propagation. The model should be as precise as possible but yet intuitive. This is why GTD is chosen in this work instead of UTD. The intuitiveness of the exponential decaying fields in GTD outweighs the precision of the UTD in the transition region. Still the model should include effects from the electromagnetic properties of the human tissue, the frequency and polarization of the electromagnetic field, as well as the curvature and torsion of the geometry. Out of consideration for the intuitiveness and from the good correlation obtained in [1], [3], [7], a homogenous model is used. This choice is further motivated by the relatively small impact of a layered model seen in [15].

The model addresses two types of propagation. The first is the off-body connection between an antenna on the body and an antenna off the body. By assuming the off-body antenna is placed in the far-field, this can be modeled by calculating the fields on an infinite long, lossy dielectric (human tissue) cylinder caused by an incident plane wave. The second is the on-body connection between two antennas placed on the body. This is modeled by calculating the fields on an infinite long, lossy dielectric (human tissue) cylinder caused by an infinitesimal dipole source on the surface of the cylinder. The cylinder is chosen since it gives rise to geodesics (shortest 


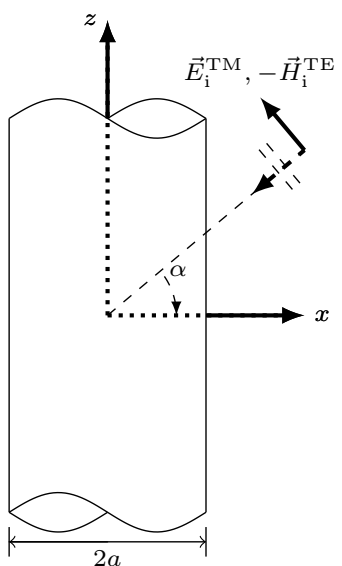

(a) Side view

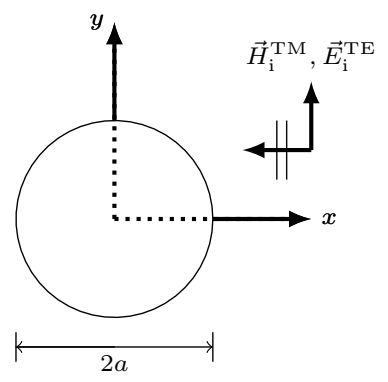

(b) Top view
Fig. 1. The geometry of the infinitely long dielectric cylinder model with an incident plane wave. The cylinder is homogenous with permittivity $\epsilon_{1}$ and permeability $\mu_{1}$.

paths) with all combinations of curvature and torsion. This makes it possible to approximate any kind of geometry by the assumption that the propagation only depends on the local geometry. Therefore, general GTD expressions for the offbody and on-body propagation can be found from these two canonical problems.

The paper is organized as follows. In Section II the eigenfunction solution to the two canonical problems are found. In Section III the asymptotic or GTD expressions are found. In Section IV, numerical calculations of the eigenfunction solution to the plane wave incidence case is compared to the asymptotic solution to validate the expression. Furthermore, the limitations of the GTD solution is investigated. In Section $\mathrm{V}$ the GTD expressions for the two cases are generalized to any geometry. Finally, Section VI contains the conclusion.

The approach followed in the paper is found in [16] which covers the topic in detail and is a good source for introduction and details. An $e^{j \omega t}$ time dependence for the electromagnetic fields are assumed and suppressed throughout the paper. The constitutive parameters of human tissue are taken from [17], [18].

\section{EIGENFUnCtion Solution}

\section{A. Plane Wave Incidence}

The solution to the canonical problem of an infinite long, lossy dielectric cylinder illuminated by a oblique incident plane wave is shown in the following. The geometry is seen in Fig. 1. A standard polar coordinate system is used, based on the coordinate system shown in the figure. The original solution can be found in [19], [20]. Note that [19] has a sign error in the coefficient named $c_{n}$ as pointed out in [9]. The problem has also previously been solved for a IBC cylinder [11]. The notation of [11] is used.

The incident $\mathrm{TM}_{\mathrm{z}}$ and $\mathrm{TE}_{\mathrm{z}}$ polarized plane wave is given by:

$$
\begin{aligned}
& \vec{E}_{\mathrm{i}}^{\mathrm{TM}}(\rho, \phi, z)=E_{0}(\hat{z} \cos \alpha-\hat{x} \sin \alpha) e^{j k_{0}(z \sin \alpha+\rho \cos \phi \cos \alpha)} \\
& \vec{E}_{\mathrm{i}}^{\mathrm{TE}}(\rho, \phi, z)=E_{0} \hat{y} e^{j k_{0}(z \sin \alpha+\rho \cos \phi \cos \alpha)}
\end{aligned}
$$

where $k_{0}=\omega \sqrt{\epsilon_{0} \mu_{0}}$ is the free space wave number with the free space permittivity and permeability given by $\epsilon_{0}$ and $\mu_{0}$, respectively. $E_{0}$ is the amplitude of the incident wave. And $\alpha$ is the angle of incidence as shown in Fig. 1.

Vector potentials for $\mathrm{TM}_{\mathrm{z}}$ and $\mathrm{TE}_{\mathrm{z}}$ polarized incident waves are introduced with the notation of [21]

- For a $\mathrm{TM}_{\mathrm{z}}$ incident wave: $\vec{A}_{\mathrm{z}}^{\mathrm{TM}}=\hat{z} A_{\mathrm{z}}^{\mathrm{TM}}, \vec{F}_{\mathrm{z}}^{\mathrm{TM}}=\hat{z} F_{\mathrm{z}}^{\mathrm{TM}}$ and

$$
\begin{aligned}
& \vec{E}_{1}=-\nabla \times \vec{F}_{\mathrm{z}}^{\mathrm{TM}}+\frac{1}{j \omega \epsilon_{0}} \nabla \times \nabla \times \vec{A}_{\mathrm{z}}^{\mathrm{TM}} \\
& \vec{H}_{1}=\nabla \times \vec{A}_{\mathrm{z}}^{\mathrm{TM}}+\frac{1}{j \omega \mu_{0}} \nabla \times \nabla \times \vec{F}_{\mathrm{z}}^{\mathrm{TM}}
\end{aligned}
$$

- For a TE $\mathrm{z}_{\mathrm{z}}$ incident wave: $\vec{F}_{\mathrm{z}}^{\mathrm{TE}}=\hat{z} F_{\mathrm{z}}^{\mathrm{TE}}, \vec{A}_{\mathrm{z}}^{\mathrm{TE}}=\hat{z} A_{\mathrm{z}}^{\mathrm{TE}}$ and

$$
\begin{aligned}
& \vec{E}_{2}=-\nabla \times \vec{F}_{\mathrm{z}}^{\mathrm{TE}}+\frac{1}{j \omega \epsilon_{0}} \nabla \times \nabla \times \vec{A}_{\mathrm{z}}^{\mathrm{TE}} \\
& \vec{H}_{2}=\nabla \times \vec{A}_{\mathrm{z}}^{\mathrm{TE}}+\frac{1}{j \omega \mu_{0}} \nabla \times \nabla \times \vec{F}_{\mathrm{z}}^{\mathrm{TE}}
\end{aligned}
$$

The total field will be a superposition of these fields:

$$
\begin{aligned}
& \vec{E}=\vec{E}_{1}+\vec{E}_{2} \\
& \vec{H}=\vec{H}_{1}+\vec{H}_{2}
\end{aligned}
$$

It can be shown that the $\mathrm{TM}_{\mathrm{z}}$ and $\mathrm{TE}_{\mathrm{z}}$ potentials satisfy the same wave equation as the fields [21]:

$$
\left(\nabla^{2}+k_{0}^{2}\right)\left[\begin{array}{c}
\vec{A}_{\mathrm{z}} \\
\vec{F}_{\mathrm{z}}
\end{array}\right]=0
$$

The approach in [19] is followed. First the $z$ variation is isolated in the term $e^{j k_{\mathrm{z}} z}$ where $k_{\mathrm{z}}=k_{0} \sin \alpha$. The incident plane wave is written with the expansion of the $e^{j \rho \cos \phi \sin \alpha}$ term by the use of first-order Bessel functions. This gives the following equations for the fields outside the cylinder:

$$
\left[\begin{array}{c}
A_{\mathrm{z}} \\
F_{\mathrm{z}}
\end{array}\right]=e^{j k_{\mathrm{z}} z} \sum_{n=-\infty}^{\infty} j^{n} e^{-j n \phi}\left[\begin{array}{c}
\tilde{A}_{\mathrm{z}} \\
\tilde{F}_{\mathrm{z}}
\end{array}\right]
$$

where $\phi$ and $z$ are the coordinates for the observation point and $\tilde{A}_{\mathrm{z}}$ and $\tilde{F}_{\mathrm{z}}$ are given by:

$$
\begin{aligned}
& {\left[\begin{array}{c}
\tilde{A}_{\mathrm{z}}^{\mathrm{TM}} \\
\tilde{F}_{\mathrm{z}}^{\mathrm{TE}}
\end{array}\right]=\left[\begin{array}{c}
C_{0}^{\mathrm{m}} \\
C_{0}^{\mathrm{e}}
\end{array}\right]\left(J_{n}\left(k_{\mathrm{t} 0} \rho\right)-\left[\begin{array}{c}
A_{n}^{\mathrm{m}} \\
A_{n}^{\mathrm{e}}
\end{array}\right] H_{n}^{(2)}\left(k_{\mathrm{t} 0} \rho\right)\right)} \\
& {\left[\begin{array}{c}
\tilde{F}_{\mathrm{z}}^{\mathrm{TM}} \\
\tilde{A}_{\mathrm{z}}^{\mathrm{TE}}
\end{array}\right]=\left[\begin{array}{c}
-C_{0}^{\mathrm{e}} \\
C_{0}^{\mathrm{m}}
\end{array}\right] A_{n}^{\mathrm{c}} H_{n}^{(2)}\left(k_{\mathrm{t} 0} \rho\right)}
\end{aligned}
$$

where $\rho$ is the coordinate of the observation point, $k_{\mathrm{t} 0}=$ $k_{0} \cos \alpha$ and $\eta_{0}=\sqrt{\frac{\mu_{0}}{\epsilon_{0}}}$ is the intrinsic impedance of free 
space, $J_{n}$ are first-order Bessel functions, $H_{n}^{(2)}$ are secondorder Hankel functions and:

$$
\begin{aligned}
C_{0}^{\mathrm{m}} & =E_{0} \frac{j}{\eta_{0} k_{\mathrm{t} 0}} \\
C_{0}^{\mathrm{e}} & =E_{0} \frac{1}{j k_{\mathrm{t} 0}}
\end{aligned}
$$

Similar expressions for the fields inside the cylinder can be found in [19], [20]. The tangential fields inside and outside the cylinder are equated at the surface of the cylinder to satisfy the boundary conditions. This gives the following coefficients:

$$
\begin{aligned}
{\left[\begin{array}{c}
A_{n}^{\mathrm{m}} \\
A_{n}^{\mathrm{e}}
\end{array}\right] } & =\frac{J_{n}\left(k_{\mathrm{t} 0} a\right)\left(\left[\begin{array}{c}
F_{n} N_{n} \\
G_{n} M_{n}
\end{array}\right]-q_{\mathrm{c}}^{2}\right)}{H_{n}^{(2)}\left(k_{\mathrm{t} 0} a\right)\left(F_{n} G_{n}-q_{\mathrm{c}}^{2}\right)} \\
A_{n}^{\mathrm{c}} & =\frac{2 q_{\mathrm{c}}}{\pi\left(k_{\mathrm{t} 0} a\right)\left(H_{n}^{(2)}\left(k_{\mathrm{t} 0} a\right)\right)^{2}\left(F_{n} G_{n}-q_{\mathrm{c}}^{2}\right)}
\end{aligned}
$$

with

$$
\begin{aligned}
& {\left[\begin{array}{c}
F_{n} \\
G_{n}
\end{array}\right]=\frac{H_{n}^{(2) \prime}\left(k_{\mathrm{t} 0} a\right)}{H_{n}^{(2)}\left(k_{\mathrm{t} 0} a\right)}-\left[\begin{array}{c}
q_{\mathrm{e}} \\
q_{\mathrm{m}}
\end{array}\right] \frac{J_{n}^{\prime}\left(k_{\mathrm{t} 1} a\right)}{J_{n}\left(k_{\mathrm{t} 1} a\right)}} \\
& {\left[\begin{array}{c}
M_{n} \\
N_{n}
\end{array}\right]=\frac{J_{n}^{\prime}\left(k_{\mathrm{t} 0} a\right)}{J_{n}\left(k_{\mathrm{t} 0} a\right)}-\left[\begin{array}{c}
q_{\mathrm{e}} \\
q_{\mathrm{m}}
\end{array}\right] \frac{J_{n}^{\prime}\left(k_{\mathrm{t} 1} a\right)}{J_{n}\left(k_{\mathrm{t} 1} a\right)}}
\end{aligned}
$$

where $k_{\mathrm{t} 1}=\sqrt{k_{1}^{2}-\left(k_{0} \sin \alpha\right)^{2}}$. The wave number of the lossy dielectric $k_{1}$ is given by $k_{1}=\omega \sqrt{\epsilon_{1} \mu_{1}}$ with the permittivity and permeability of the lossy dielectric given by $\epsilon_{1}$ and $\mu_{1}$, respectively, and finally,

$$
\begin{aligned}
q_{\mathrm{m}} & =\frac{\epsilon_{1} k_{\mathrm{t} 0}}{\epsilon_{0} k_{\mathrm{t} 1}} \\
q_{\mathrm{e}} & =\frac{\mu_{1} k_{\mathrm{t} 0}}{\mu_{0} k_{\mathrm{t} 1}} \\
q_{\mathrm{c}} & =\frac{n k_{\mathrm{z}}}{k_{\mathrm{t} 0} k_{0} a}\left[1-\left(\frac{k_{\mathrm{t} 0}}{k_{\mathrm{t} 1}}\right)^{2}\right]
\end{aligned}
$$

\section{B. Infinitesimal Dipole Sources}

The canonical problem of the fields from an infinitesimal dipole source on the surface of an infinitely long dielectric cylinder is solved in the following. The focus is on the case with the observation point placed on the surface of the cylinder as well. This makes it possible to calculate the coupling or path loss between two infinitesimal dipoles on the surface. The geometry is defined in Fig. 2. Again a standard polar coordinate system is used. Without loss of generalization, the source point is assumed to be at $(x=a, y=0, z=0)$. The location of the observation point is then simply given by its coordinates. A similar approach for the impedance boundary conditions has been made in [12], [22], [23] and for a perfect electric conductor in [24]-[26]. The case with the source away from the cylinder is solved in [27]. The same potentials as

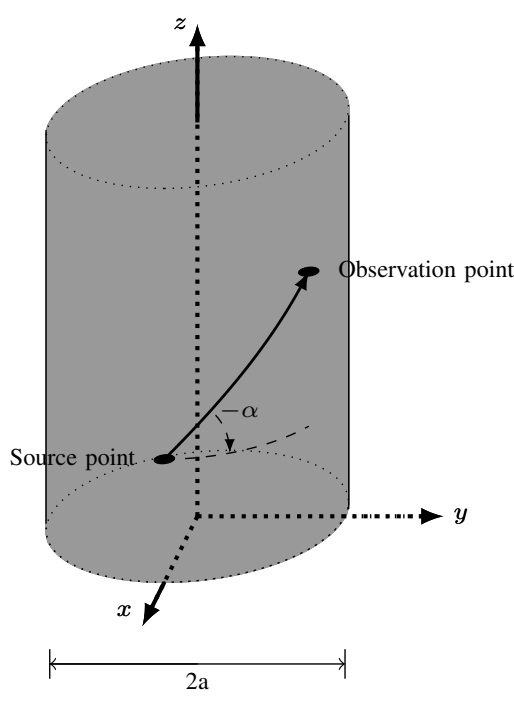

Fig. 2. The geometry of the infinitely long dielectric cylinder model with a source and observation point. The source and observation points are connected by the shortest path between them. The cylinder is homogenous with permittivity $\epsilon_{1}$ and permeability $\mu_{1}$.

defined in Eq. 2 and Eq. 3 are used. Furthermore, the following Fourier transforms of the potentials are defined:

$$
\begin{aligned}
& A_{\mathrm{z}}=\frac{1}{2 \pi} \sum_{n=-\infty}^{\infty} e^{j n \phi} \int_{-\infty}^{\infty} \tilde{A}_{\mathrm{z}} e^{j k_{\mathrm{z}} z} d k_{\mathrm{z}} \\
& F_{\mathrm{z}}=\frac{1}{2 \pi} \sum_{n=-\infty}^{\infty} e^{j n \phi} \int_{-\infty}^{\infty} \tilde{F}_{\mathrm{z}} e^{j k_{\mathrm{z}} z} d k_{\mathrm{z}}
\end{aligned}
$$

where $\phi$ and $z$ are the coordinates of the observation point.

By solving the boundary condition on the cylinder for the tangential fields, the potentials from tangential magnetic and electric infinitesimal dipoles can be found. The potentials from the z-oriented sources become:

$$
\begin{aligned}
\tilde{A}_{\mathrm{z}}= & \frac{H_{n}^{(2)}\left(k_{\mathrm{t} 0} \rho\right)}{2 \pi a k_{\mathrm{t} 0}\left(F_{n} G_{n}-q_{\mathrm{c}}^{2}\right) H_{n}^{(2)}\left(k_{\mathrm{t} 0} a\right)} \\
& \cdot\left(-F_{n} J_{\mathrm{z}}-\frac{j q_{\mathrm{c}}}{\eta_{0}} M_{\mathrm{z}}\right) \\
\tilde{F}_{\mathrm{z}}= & \frac{H_{n}^{(2)}\left(k_{\mathrm{t} 0} \rho\right)}{2 \pi a k_{\mathrm{t} 0}\left(F_{n} G_{n}-q_{\mathrm{c}}^{2}\right) H_{n}^{(2)}\left(k_{\mathrm{t} 0} a\right)} \\
& \cdot\left(j \eta_{0} q_{\mathrm{c}} J_{\mathrm{z}}-G_{n} M_{\mathrm{z}}\right)
\end{aligned}
$$

where $\rho$ is the radial coordinate of the observation point, which will be equal to $a$ in our case. $J_{\mathrm{z}}$ and $M_{\mathrm{z}}$ are the z-oriented electric and magnetic dipole moments, respectively. And the 
potentials from the $\phi$-oriented sources are:

$$
\begin{aligned}
& \tilde{A}_{\mathrm{z}}= \frac{H_{n}^{(2)}\left(k_{\mathrm{t} 0} \rho\right)}{2 \pi a k_{\mathrm{t} 0}\left(F_{n} G_{n}-q_{\mathrm{c}}^{2}\right) H_{n}^{(2)}\left(k_{\mathrm{t} 0} a\right)} \\
& \cdot\left[\left(\frac{k_{\mathrm{z}} n H_{n}^{(2) \prime}\left(k_{\mathrm{t} 0} a\right)}{k_{\mathrm{t} 0} a k_{\mathrm{t} 1}^{2} H_{n}^{(2)}\left(k_{\mathrm{t} 0} a\right)}-\frac{q_{\mathrm{e}} k_{\mathrm{z}} n J_{n}{ }^{\prime}\left(k_{\mathrm{t} 0} a\right)}{k_{\mathrm{t} 0}^{3} a J_{n}\left(k_{\mathrm{t} 0} a\right)}\right) J_{\phi}\right. \\
&\left.-\left(\frac{j k_{1} J_{n}^{\prime}\left(k_{\mathrm{t} 0} a\right)}{k_{\mathrm{t} 1} \eta_{1} J_{n}\left(k_{\mathrm{t} 0} a\right)} F_{n}-\frac{j n k_{\mathrm{z}}}{\eta_{0} k_{\mathrm{t} 1}^{2} a} q_{\mathrm{c}}\right) M_{\phi}\right] \\
& \tilde{F}_{\mathrm{z}}= \frac{H_{n}^{(2)}\left(k_{\mathrm{t} 0} \rho\right)}{2 \pi a k_{\mathrm{t} 0}\left(F_{n} G_{n}-q_{\mathrm{c}}^{2}\right) H_{n}^{(2)}\left(k_{\mathrm{t} 0} a\right)} \\
& \cdot\left[\left(\frac{j k_{1} \eta_{1} J_{n}{ }^{\prime}\left(k_{\mathrm{t} 0} a\right)}{k_{\mathrm{t} 1} J_{n}\left(k_{\mathrm{t} 0} a\right)} G_{n}-\frac{j \eta_{0} n k_{\mathrm{z}}}{k_{\mathrm{t} 1}^{2} a} q_{\mathrm{c}}\right) J_{\phi}\right. \\
&\left.+\left(\frac{k_{\mathrm{z}} n H_{n}^{(2) \prime}\left(k_{\mathrm{t} 0} a\right)}{k_{\mathrm{t} 0} a k_{\mathrm{t} 1}^{2} H_{n}^{(2)}\left(k_{\mathrm{t} 0} a\right)}-\frac{q_{\mathrm{m}} k_{\mathrm{z}} n J_{n}{ }^{\prime}\left(k_{\mathrm{t} 0} a\right)}{k_{\mathrm{t} 0}^{3} a J_{n}\left(k_{\mathrm{t} 0} a\right)}\right) M_{\phi}\right]
\end{aligned}
$$

where $J_{\phi}$ and $M_{\phi}$ are the $\phi$-oriented electric and magnetic dipole moments, respectively. In the potentials the symbols are defined as for the plane wave incidence, except for $k_{\mathrm{z}}$ that is a variable here. This also effects $k_{\mathrm{t} 0}$ and $k_{\mathrm{t} 1}$, which will be given as $k_{\mathrm{t} 0}=\sqrt{k_{0}^{2}-k_{\mathrm{z}}^{2}}$ and $k_{\mathrm{t} 1}=\sqrt{k_{1}^{2}-k_{\mathrm{z}}^{2}}$. The definition of $q_{\mathrm{m}}, q_{\mathrm{e}}$ and $q_{\mathrm{c}}$ are the same, but the updated expresions for $k_{\mathrm{t} 0}, k_{\mathrm{t} 1}$ and $k_{\mathrm{z}}$ should be used.

The potentials from the magnetic and electric infinitesimal dipoles oriented normal to the surface of the cylinder can be found by the use of the reciprocity theorem and the fields from the z-oriented sources as shown here:

$$
\begin{aligned}
& E_{\rho}^{J_{\mathrm{z}}} J_{\rho}=\quad E_{\mathrm{z}}^{J_{\rho}} J_{\mathrm{z}}=\quad \frac{1}{j \omega \epsilon_{0}} k_{\mathrm{t} 0}^{2} A_{\mathrm{z}}^{J_{\rho}} J_{\mathrm{z}} \\
& E_{\rho}^{M_{\mathrm{z}}} J_{\rho}=-H_{\mathrm{z}}^{J_{\rho}} M_{\mathrm{z}}=-\frac{1}{j \omega \mu_{0}} k_{\mathrm{t} 0}^{2} F_{z}^{J_{\rho}} M_{\mathrm{z}} \\
& H_{\rho}^{J_{\mathrm{z}}} M_{\rho}=-E_{\mathrm{z}}^{M_{\rho}} J_{\mathrm{z}}=-\frac{1}{j \omega \epsilon_{0}} k_{\mathrm{t} 0}^{2} A_{\mathrm{z}}^{M_{\rho}} J_{\mathrm{z}} \\
& H_{\rho}^{M_{\mathrm{z}}} M_{\rho}=\quad H_{\mathrm{z}}^{M_{\rho}} M_{\mathrm{z}}=\frac{1}{j \omega \mu_{0}} k_{\mathrm{t} 0}^{2} F_{\mathrm{z}}^{M_{\rho}} M_{\mathrm{z}}
\end{aligned}
$$

The potentials from the $\rho$-oriented sources become:

$$
\begin{aligned}
\tilde{A}_{\mathrm{z}}= & \frac{H_{n}^{(2)}\left(k_{\mathrm{t} 0} \rho\right)}{2 \pi a k_{\mathrm{t} 0}\left(F_{n} G_{n}-q_{\mathrm{c}}^{2}\right) H_{n}^{(2)}\left(k_{\mathrm{t} 0} a\right)} \\
& \cdot\left[\left(\frac{j n k_{0}}{k_{\mathrm{t} 0}^{2} a} q_{\mathrm{c}}-\frac{j k_{\mathrm{z}} H_{n}^{(2) \prime}\left(k_{\mathrm{t} 0} a\right)}{k_{\mathrm{t} 0} H_{n}^{(2)}\left(k_{\mathrm{t} 0} a\right)} F_{n}\right) J_{\rho}\right. \\
& \left.-\left(\frac{n k_{0}}{k_{\mathrm{t} 0}^{2} a \eta_{0}} F_{n}-\frac{k_{\mathrm{z}} H_{n}^{(2) \prime}\left(k_{\mathrm{t} 0} a\right)}{k_{\mathrm{t} 0} \eta_{0} H_{n}^{(2)}\left(k_{\mathrm{t} 0} a\right)} q_{\mathrm{c}}\right) M_{\rho}\right] \\
\tilde{F}_{\mathrm{z}}= & \frac{H_{n}^{(2)}\left(k_{\mathrm{t} 0} \rho\right)}{2 \pi a k_{\mathrm{t} 0}\left(F_{n} G_{n}-q_{\mathrm{c}}^{2}\right) H_{n}^{(2)}\left(k_{\mathrm{t} 0} a\right)} \\
& \cdot\left[\left(\frac{n k_{0} \eta_{0}}{k_{\mathrm{t} 0}^{2} a} G_{n}-\frac{k_{\mathrm{z}} \eta_{0} H_{n}^{(2) \prime}\left(k_{\mathrm{t} 0} a\right)}{k_{\mathrm{t} 0} H_{n}^{(2)}\left(k_{\mathrm{t} 0} a\right)} q_{\mathrm{c}}\right) J_{\rho}\right. \\
& \left.+\left(\frac{j n k_{0}}{k_{\mathrm{t} 0}^{2} a} q_{\mathrm{c}}-\frac{j k_{\mathrm{z}} H_{n}^{(2) \prime}\left(k_{\mathrm{t} 0} a\right)}{k_{\mathrm{t} 0} H_{n}^{(2)}\left(k_{\mathrm{t} 0} a\right)} G_{n}\right) M_{\rho}\right]
\end{aligned}
$$

where $J_{\rho}$ and $M_{\rho}$ are the $\rho$-oriented electric and magnetic dipole moments, respectively.

\section{GTD SOLUTION}

\section{A. Plane Wave Incidence}

To evaluate Eq. 7 an assumption is made to simplify the fractions $\frac{J_{n}^{\prime}\left(k_{\mathrm{t} 1} a\right)}{J_{n}\left(k_{\mathrm{t} 1} a\right)}$. By the use of the Debye representation of the Bessel function given in [28] the fractions can be rewritten as:

$$
\begin{aligned}
\frac{J_{n}^{\prime}\left(k_{\mathrm{t} 1} a\right)}{J_{n}\left(k_{\mathrm{t} 1} a\right)} & \approx \sqrt{1-\left(\frac{n}{k_{\mathrm{t} 1} a}\right)^{2}} \\
\cdot \tan \left(-k_{\mathrm{t} 1}\right. & \left.a \sqrt{1-\left(\frac{n}{k_{\mathrm{t} 1} a}\right)^{2}}-j n \operatorname{acosh}\left(\frac{n}{k_{\mathrm{t} 1} a}\right)+\frac{\pi}{4}\right) \\
& \approx j \sqrt{1-\left(\frac{n}{k_{\mathrm{t} 1} a}\right)^{2}}
\end{aligned}
$$

The first approximation is valid for large $n$ and $k_{\mathrm{t} 1} a$, but is good for small $n$ as well. The second is valid when the imaginary part of the argument of the tangent function is large. This is the case when the cylinder is opaque, which leads to a large imaginary part of $k_{\mathrm{t} 1} a$.

By applying the Watson transformation [29] to Eq. 7 with the approximation of Eq. 17 the potentials can be calculated in the deep shadow by the use of the Cauchy residue theorem. Furthermore, the following Fock substitution has been applied [30]:

$$
v=k_{\mathrm{t} 0} a+m_{\mathrm{t}} \tau
$$

with $m_{\mathrm{t}}=\left(k_{\mathrm{t} 0} a / 2\right)^{1 / 3}$. The Hankel functions have been approximated by the use of the Fock-Airy functions $W_{1,2}(\tau)$. This is a valid approximation when the order and argument are approximately equivalent. This results in the following expressions for the potentials:

$$
\begin{aligned}
{\left[\begin{array}{c}
A_{\mathrm{z}}^{\mathrm{TM}} \\
F_{\mathrm{z}}^{\mathrm{TE}}
\end{array}\right]^{ \pm} } & \sim \sum_{p=1}^{\infty}\left[\begin{array}{c}
C_{0}^{\mathrm{m}} \\
C_{0}^{\mathrm{e}}
\end{array}\right]\left[\begin{array}{c}
D_{p}^{\mathrm{m}} \\
D_{p}^{\mathrm{e}}
\end{array}\right] \sqrt{\frac{k_{0} \pi}{2 j}} \cos \alpha H_{v}^{(2)}\left(k_{\mathrm{t} 0} \rho\right) \\
& \cdot e^{-j k_{0} t \cos ^{2} \alpha-\alpha_{p} t} \\
{\left[\begin{array}{c}
F_{\mathrm{z}}^{\mathrm{TM}} \\
A_{\mathrm{z}}^{\mathrm{TE}}
\end{array}\right]^{ \pm} } & \sim \sum_{p=1}^{\infty}\left[\begin{array}{c}
-C_{0}^{\mathrm{e}} \\
C_{0}^{\mathrm{m}}
\end{array}\right] D_{p}^{\mathrm{c}} \sqrt{\frac{k_{0} \pi}{2 j}} \cos \alpha H_{v}^{(2)}\left(k_{\mathrm{t} 0} \rho\right) \\
& \cdot e^{-j k_{0} t \cos ^{2} \alpha-\alpha_{p} t}
\end{aligned}
$$

where $t$ is the distance the rays travel on the surface of the cylinder. The \pm refers to the direction of travel. The '+' corresponds to counter-clockwise travel where $t=\frac{(\phi-\pi / 2) a}{\cos \alpha}$. The '-' corresponds to clockwise travel where $t=\frac{(3 \pi / 2-\phi) a}{\cos \alpha}$. Multiple encirclements can be included by dividing with $1-e^{-j k_{\mathrm{t} 0} 2 \pi a-\alpha_{p} 2 \pi a / \cos \alpha}$, which for all relevant situations is approximately equal to 1 . The diffraction constants are given by:

$$
\begin{aligned}
& D_{p}^{\mathrm{m}, \mathrm{e}}=\frac{2 \pi m_{\mathrm{t}}^{2}\left(\frac{-W_{2}^{\prime}(\tau)}{m_{\mathrm{t}} W_{2}(\tau)}-j q_{\mathrm{e}, \mathrm{m}} \sqrt{1-\left(\frac{k_{\mathrm{t} o} a+m_{\mathrm{t}} \tau_{p}}{k_{\mathrm{t} 1} a}\right)^{2}}\right)}{\sqrt{\frac{k_{0} \pi}{2 j}} \cos \alpha D_{\mathrm{W}}^{\prime}\left(\tau_{p}\right)} \\
& D_{p}^{\mathrm{c}}= \pm \frac{2 j \pi m_{\mathrm{t}}^{2} q_{c}\left(\tau_{p}\right)}{\sqrt{\frac{k_{0} \pi}{2 j}} \cos \alpha D_{\mathrm{W}}^{\prime}\left(\tau_{p}\right)}= \pm \sqrt{-D_{p}^{\mathrm{m}} D_{p}^{\mathrm{e}}}
\end{aligned}
$$


where $\tau_{p}$ is the $p^{\text {th }}$ singularity of $A_{n}^{\mathrm{m}}, A_{n}^{\mathrm{e}}$, and $A_{n}^{\mathrm{c}}$ given by the $p^{\text {th }}$ zero of

$$
\begin{aligned}
D_{\mathrm{W}}(\tau) & =\left(W_{2}^{\prime}(\tau)+j m_{\mathrm{t}} q_{\mathrm{e}} \sqrt{1-\left(\frac{k_{\mathrm{t} 0} a+m_{\mathrm{t}} \tau}{k_{\mathrm{t} 1} a}\right)^{2}} W_{2}(\tau)\right) \\
& \cdot\left(W_{2}^{\prime}(\tau)+j m_{\mathrm{t}} q_{\mathrm{m}} \sqrt{1-\left(\frac{k_{\mathrm{t} 0} a+m_{\mathrm{t}} \tau}{k_{\mathrm{t} 1} a}\right)^{2}} W_{2}(\tau)\right) \\
& -\left[m_{\mathrm{t}} q_{\mathrm{c}}(\tau) W_{2}(\tau)\right]^{2}
\end{aligned}
$$

and $D_{\mathrm{W}}^{\prime}(\tau)$ is the derivative of $D_{\mathrm{W}}(\tau)$ with respect to $\tau$. This can, at $\tau_{p}$, be expressed as

$$
\begin{aligned}
& D_{\mathrm{W}}^{\prime}\left(\tau_{p}\right)=\left(\tau_{p} W_{2}\left(\tau_{p}\right)^{2}-\frac{j m_{\mathrm{t}}^{2} q_{\mathrm{e}} \frac{k_{\mathrm{t} 0} a+m_{\mathrm{t}} \tau_{p}}{\left(k_{\mathrm{t} 1} a\right)^{2}}}{\sqrt{1-\left(\frac{k_{\mathrm{t} 0} a+m_{\mathrm{t}} \tau_{p}}{k_{\mathrm{t} 1} a}\right)^{2}}} W_{2}\left(\tau_{p}\right)^{2}\right. \\
& \left.-W_{2}^{\prime}\left(\tau_{p}\right)^{2}\right)\left(\frac{W_{2}^{\prime}\left(\tau_{p}\right)}{W_{2}\left(\tau_{p}\right)}+j m_{\mathrm{t}} q_{\mathrm{m}} \sqrt{1-\left(\frac{k_{\mathrm{t} 0} a+m_{\mathrm{t}} \tau_{p}}{k_{\mathrm{t} 1} a}\right)^{2}}\right) \\
& +\left(\tau_{p} W_{2}\left(\tau_{p}\right)^{2}-\frac{j m_{\mathrm{t}}^{2} q_{\mathrm{m}} \frac{k_{\mathrm{t} 0} a+m_{\mathrm{t}} \tau_{p}}{\left(k_{\mathrm{t} 1} a\right)^{2}}}{\sqrt{1-\left(\frac{k_{\mathrm{t} 0} a+m_{\mathrm{t}} \tau_{p}}{k_{\mathrm{t} 1} a}\right)^{2}}} W_{2}\left(\tau_{p}\right)^{2}\right. \\
& \left.-W_{2}^{\prime}\left(\tau_{p}\right)^{2}\right)\left(\frac{W_{2}^{\prime}\left(\tau_{p}\right)}{W_{2}\left(\tau_{p}\right)}+j m_{\mathrm{t}} q_{\mathrm{e}} \sqrt{1-\left(\frac{k_{\mathrm{t} 0} a+m_{\mathrm{t}} \tau_{p}}{k_{\mathrm{t} 1} a}\right)^{2}}\right) \\
& -2 m_{\mathrm{t}}^{2} q_{\mathrm{c}}\left(\tau_{p}\right) q_{\mathrm{c}}^{\prime}\left(\tau_{p}\right) W_{2}\left(\tau_{p}\right)^{2}
\end{aligned}
$$

where the Airy differential equation $W_{2}^{\prime \prime}(\tau)-\tau W_{2}(\tau)=0$ has been used to simplify the expression. In Eq. $20 \mathrm{~b}$ the right branch of the square root has to be chosen to ensure that the equal sign is correct. By substitution of $n$ with $v$ and then with $k_{\mathrm{t} 0} a+m_{\mathrm{t}} \tau$ the expressions for $q_{\mathrm{c}}(\tau)$ and $q_{\mathrm{c}}^{\prime}(\tau)$ can be found as:

$$
\begin{array}{r}
q_{\mathrm{c}}(\tau)=\left(1+\frac{\tau}{2 m_{\mathrm{t}}^{2}}\right) \frac{k_{\mathrm{z}}}{k_{0}}\left[1-\left(\frac{k_{\mathrm{t} 0}}{k_{\mathrm{t} 1}}\right)^{2}\right] \\
q_{\mathrm{c}}^{\prime}(\tau)=\frac{1}{2 m_{\mathrm{t}}^{2}} \frac{k_{\mathrm{z}}}{k_{0}}\left[1-\left(\frac{k_{\mathrm{t} 0}}{k_{\mathrm{t} 1}}\right)^{2}\right]
\end{array}
$$

The attenuation factor is given by

$$
\alpha_{p}=j \frac{m_{\mathrm{t}} \cos \alpha}{a} \tau_{p}
$$

The $\tau_{p}$ 's are found to be approximately located along the line, which originates in origo and makes an angle of $-60^{\circ}$ with the real axis in the complex plane. Therefore, the $\alpha_{p}$ 's will have an imaginary part, which will effectively increase the wave number. The wavelength along the cylinder is therefore shorter than the free space wavelength.

\section{B. Infinitesimal Dipole Sources}

The same procedure is followed as for the case of plane wave incidence. The expressions for the potentials are simplified by the use of Eq. 17. The Watson transformation is used
TABLE I

CONSTITUTIVE PARAMETERS OF DIFFERENT TISSUES

\begin{tabular}{lrrrrr}
\hline \multirow{2}{*}{ Tissue } & \multicolumn{2}{c}{$2.45 \mathrm{GHz}$} & & \multicolumn{2}{c}{$5.8 \mathrm{GHz}$} \\
\cline { 2 - 3 } \cline { 5 - 6 } & $\sigma(\mathrm{S} / \mathrm{m})$ & $\epsilon_{r}$ & & $\sigma(\mathrm{S} / \mathrm{m})$ & $\epsilon_{r}$ \\
\hline \hline Muscle [17] & 1.74 & 52.7 & & 4.96 & 48.5 \\
Skin [17] & 1.46 & 38.0 & & 3.72 & 35.1 \\
Fat [17] & 0.105 & 5.28 & & 0.293 & 4.95 \\
Standard [18] & 1.8 & 39.2 & & 5.27 & 35.3 \\
\hline
\end{tabular}

as well as the Fock substitution from Eq. 18. The integral over $k_{\mathrm{z}}$ is evaluated by the use of the steepest decent method where only the first order approximation in the asymptotic expansion is used. The details of this process are well outlined in [31]. The result for the potentials caused by a $\rho$-oriented electric source are given by:

$$
\begin{aligned}
A_{\mathrm{z}}= & J_{\rho} \sqrt{\frac{k_{0} \pi}{2 j}} \cos \alpha \sqrt{\frac{\pi}{2 j k_{0} t}} \frac{j k_{0}}{4 \pi} H_{v}^{(2)}\left(k_{\mathrm{t} 0} \rho\right) e^{-j k_{0} t-\alpha_{p} t} \\
& \cdot\left(\frac{j v}{k_{\mathrm{t} 0} a} D_{p}^{\mathrm{c}} H_{v}^{(2)}\left(k_{\mathrm{t} 0} a\right)+\sin (\alpha) D_{p}^{\mathrm{m}} H_{v}^{(2) \prime}\left(k_{\mathrm{t} 0} a\right)\right) \\
F_{\mathrm{z}}= & J_{\rho} \sqrt{\frac{k_{0} \pi}{2 j}} \cos \alpha \sqrt{\frac{\pi}{2 j k_{0} t}} \frac{j k_{0} \eta_{0}}{4 \pi} H_{v}^{(2)}\left(k_{\mathrm{t} 0} \rho\right) e^{-j k_{0} t-\alpha_{p} t} \\
& \cdot\left(\frac{j v}{k_{\mathrm{t} 0} a} D_{p}^{\mathrm{e}} H_{v}^{(2)}\left(k_{\mathrm{t} 0} a\right)-\sin (\alpha) D_{p}^{\mathrm{c}} H_{v}^{(2) \prime}\left(k_{\mathrm{t} 0} a\right)\right)
\end{aligned}
$$

The expressions for the electric and magnetic sources of other orientations have been left out in order to reduce the paper length.

From the potentials the $\rho$-oriented electric field from a $\rho$ oriented electric source is calculated and results in:

$$
\begin{aligned}
E_{\rho}= & J_{\rho} \frac{k_{0} \pi}{2 j} \cos ^{2} \alpha \frac{j k_{0} \eta_{0}}{4 \pi} \frac{e^{-j k_{0} t-\alpha_{p} t}}{\sqrt{t}} \\
& {\left[H_{v}^{(2)}\left(k_{\mathrm{t} 0} \rho\right)\right.} \\
& \cdot\left(\frac{v^{2}}{k_{\mathrm{t} 0}^{2} a \rho} D_{p}^{\mathrm{e}} H_{v}^{(2)}\left(k_{\mathrm{t} 0} a\right)+\frac{j v \sin \alpha}{k_{\mathrm{t} 0} \rho} D_{p}^{\mathrm{c}} H_{v}^{(2) \prime}\left(k_{\mathrm{t} 0} a\right)\right) \\
& +H_{v}^{(2) \prime}\left(k_{\mathrm{t} 0} \rho\right) \\
& \left.\cdot\left(\frac{j v \sin \alpha}{k_{\mathrm{t} 0} a} D_{p}^{\mathrm{c}} H_{v}^{(2)}\left(k_{\mathrm{t} 0} a\right)+\sin ^{2} \alpha D_{p}^{\mathrm{m}} H_{v}^{(2) \prime}\left(k_{\mathrm{t} 0} a\right)\right)\right]
\end{aligned}
$$

\section{NumERICAL RESUlTS}

The eigenfunction solution for the plane wave incidence given in Section II-A has been evaluated numerically. This is done by truncating the summation of Eq. 6. Truncation at $n=k_{0} \rho+20$ yields a sufficient accuracy.

The GTD solution found in Section III-A has also been evaluated. The most challenging part of the evaluation is to find the zeros of Eq. 21. This can be done by different numerical methods as listed in [11]. Here the zeros have been found by a simple algorithm that searches an area around origo in the complex plane to find minimums of error of Eq. 21. When the requested amount of minimums have been found, 


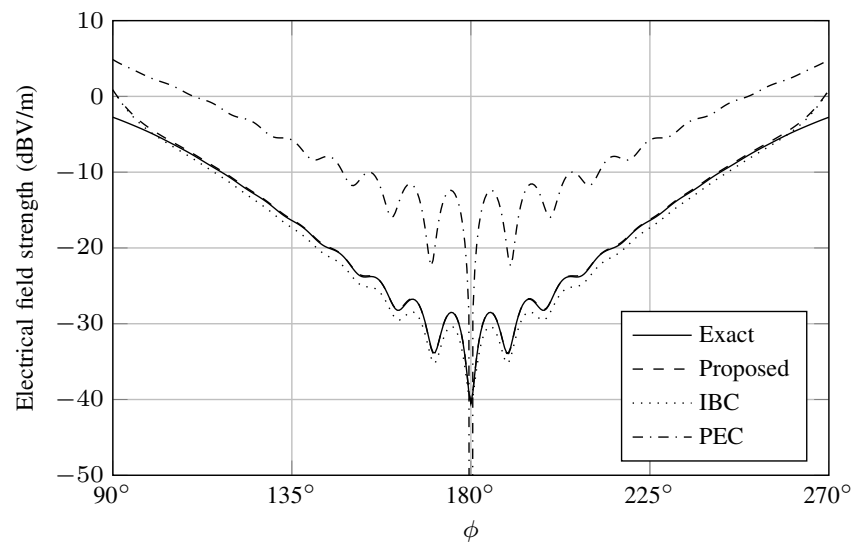

(a)

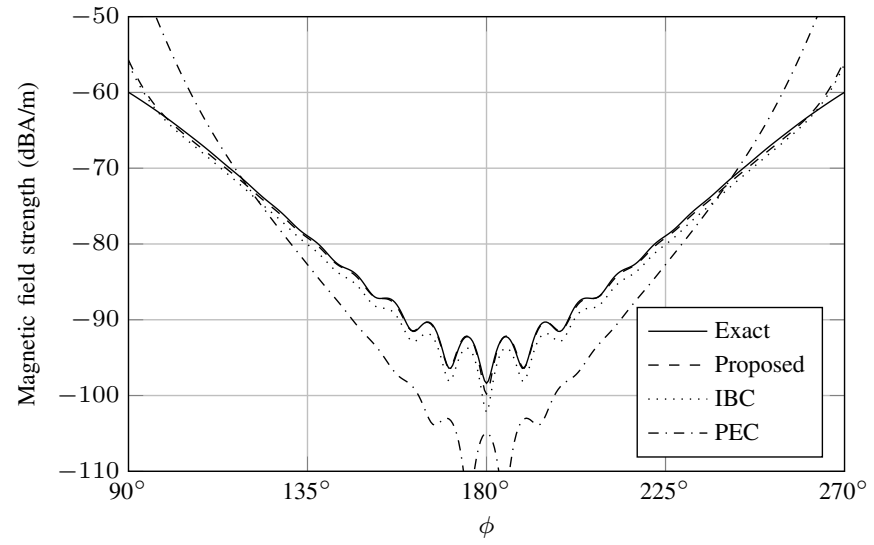

(b)

Fig. 3. Field plots for a 'fat' cylinder with $a=160 \mathrm{~mm}$ and $\alpha=30^{\circ}$ at $5.8 \mathrm{GHz}$. Electric field strength in the TE case (a) and magnetic field strength in the TM case (b).

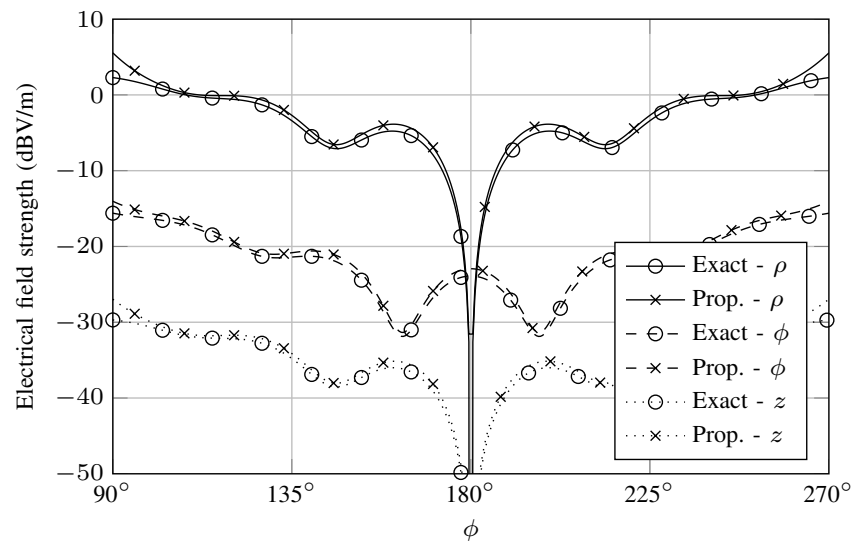

(a)

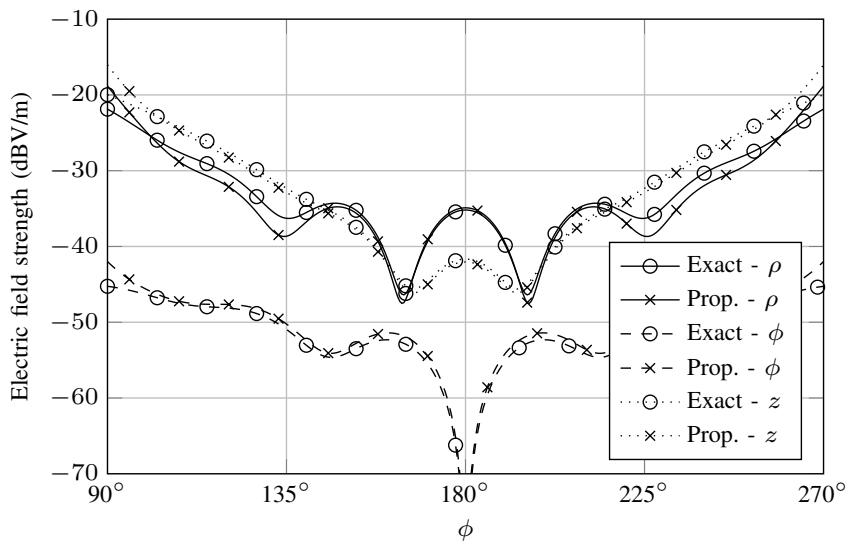

(b)

Fig. 4. Field plots for a 'human standard' cylinder with $a=80 \mathrm{~mm}$ and $\alpha=10^{\circ}$ at $2.45 \mathrm{GHz}$. Electric field strength for each polarization in TE (a) and TM (b) case.

the error for each minimum is reduced below a threshold by zooming in on each of these. The minimums are then considered to be solutions to the equation. Here the number of zeroes or modes used is four. Two modes are more than enough in the deep shadow. The error threshold is set to $10^{-8}$, but less will do.

In Fig. 3 the proposed approximation has been compared to the exact solution, an IBC approximation and a PEC approximation. The results shown are from a cylinder with radius $a=160 \mathrm{~mm}$, incidence angle of $\alpha=30^{\circ}$ and constitutive parameters of fat (see Table I). This could be a helix shaped path around the human torso. The solution frequency is $5.8 \mathrm{GHz}$. The results for the E-fields on the cylinder from a TE incident wave is seen in Fig. 3a. The results for the $\mathrm{H}$-fields on the cylinder from a TM incident wave is seen in Fig. 3b. The H-field has been chosen for the TM wave to enable comparison to the PEC approximation. The E-field on the cylinder in the TM case for a PEC cylinder is zero.

It is clear that the PEC approximation is far from precise. The IBC approximation and proposed approximation are both very close to the exact solution. In the vicinity of the shadow boundary at $90^{\circ}$ and $270^{\circ}$ they start to drift away from the exact solution as expected. The proposed solution can hardly be distinguished from the exact solution in the deep shadow. The error is less than $0.2 \mathrm{~dB}$. The IBC approximation has a small visible error, but below $2 \mathrm{~dB}$.

In Fig. 4 the results of the proposed approximation is compared to the exact solution for each of the three polarizations for each of the incident waves. The results are for a cylinder with radius $a=80 \mathrm{~mm}$, incidence angle of $\alpha=10^{\circ}$, and constitutive parameters as specified by the standard [18] (see Table I). This corresponds to a typical path on the human head. The solution frequency is $2.45 \mathrm{GHz}$. All polarizations are seen to be modeled well with less than $1 \mathrm{~dB}$ of error in the deep shadow. The errors are a bit larger than the results for the $160 \mathrm{~mm}$ cylinder at $5.8 \mathrm{GHz}$ since the electrical length of the $80 \mathrm{~mm}$ cylinder at $2.45 \mathrm{GHz}$ is close to the limit of an electrically large structure.

To investigate the area of validity for the proposed approximation, the relative root-mean-square error for a sweep of the radius of the cylinder $a$ and the angle of incidence $\alpha$ has been 


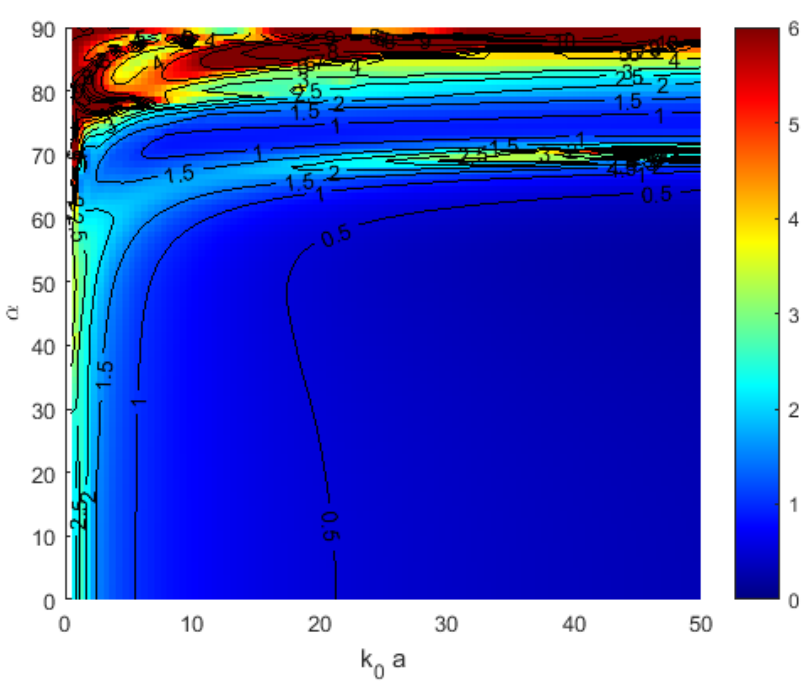

(a)

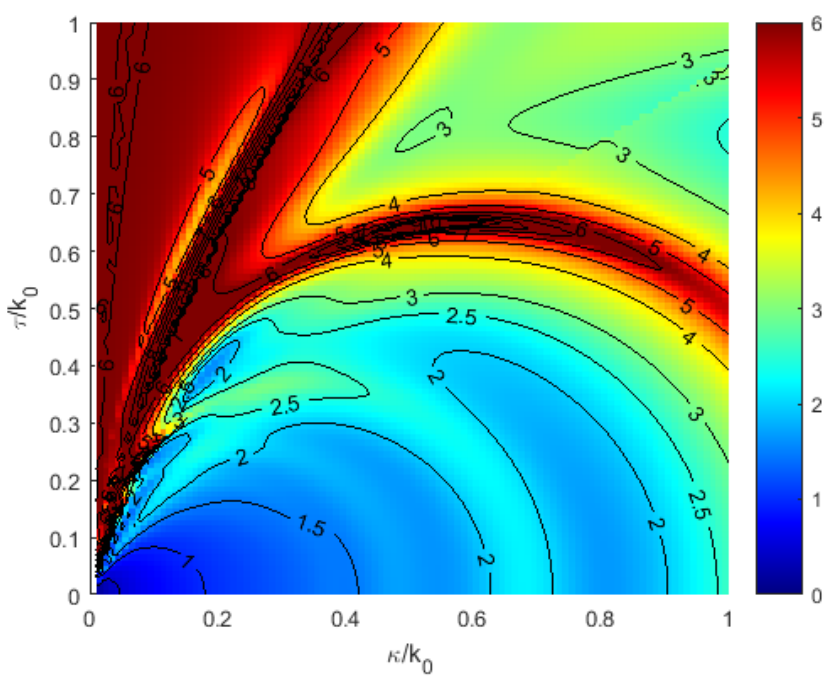

(c)

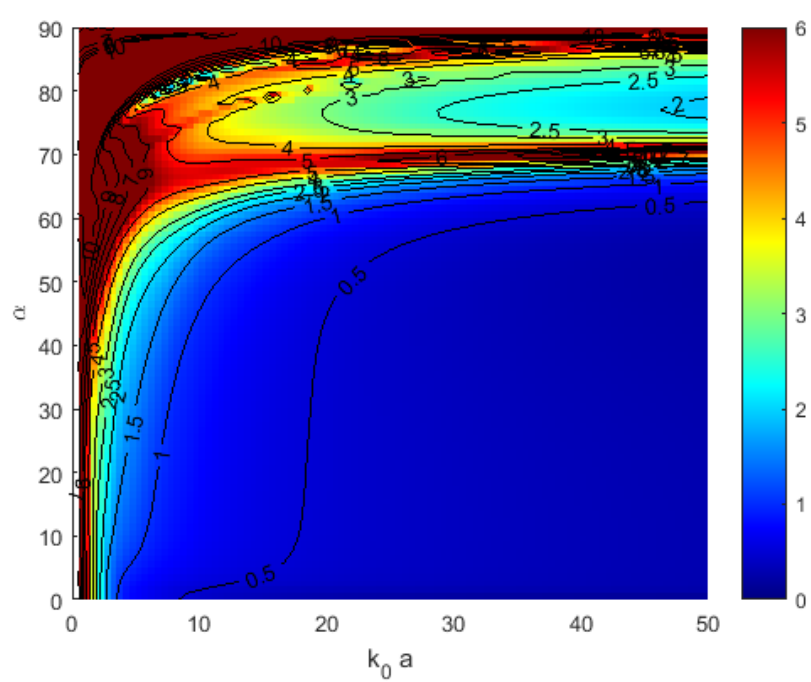

(b)

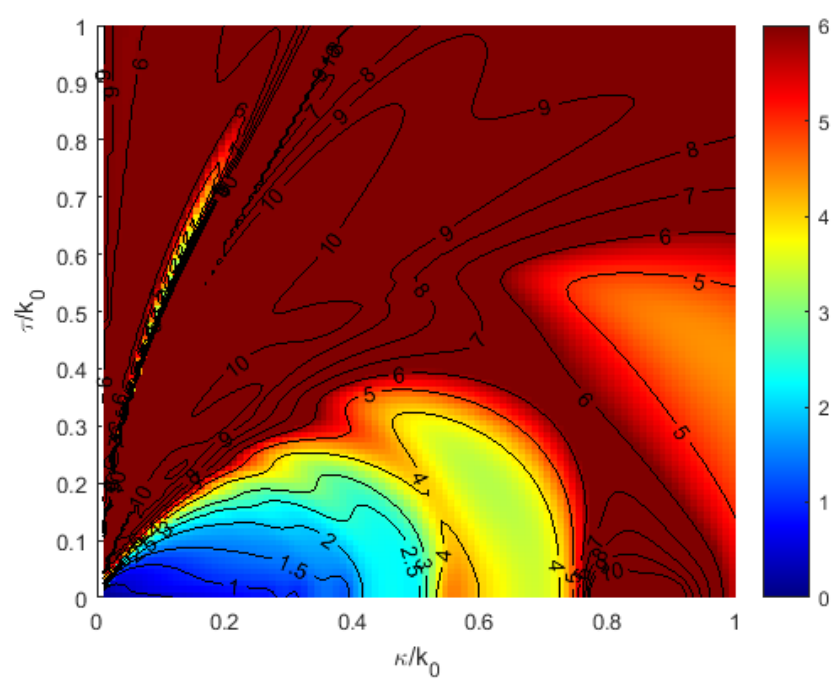

(d)

Fig. 5. Root-mean-square of the relative error (from zero (blue) to six (red) decibel) in the electric field in the deep shadow for different cylinders. As a function of the electrical length of the cylinder $k_{0} a$ and the angle of incidence $\alpha$ in the TE (a) and TM (b) cases. As a function of the electrical curvature $\frac{\kappa}{k_{0}}$ and the electrical torsion $\frac{\tau}{k_{0}}$ in the TE (c) and TM (d) cases.

calculated. The relative error $R E$ was calculated as:

$$
R E=\frac{\left|\vec{E}_{\text {exact }}-\vec{E}_{\text {approx }}\right|}{\left|\vec{E}_{\text {exact }}\right|}
$$

The relative root-mean-square error was found for the $\phi$-angles from $135^{\circ}$ to $225^{\circ}$. This is the area where all points are at least $45^{\circ}$ into the shadow. The cylinder's constitutive parameters were $\epsilon_{r}=39.2$ and $\sigma=1.8 \mathrm{~S} / \mathrm{m}$ as given by the standard [18] for $2.45 \mathrm{GHz}$. The solution frequency was $2.45 \mathrm{GHz}$. The decibel value of the RMS-error is shown in Fig. 5. In Fig. $5 \mathrm{a}$ and Fig. 5b the error is plotted for the TE and TM cases versus the electrical radius of the cylinder $k_{0} a$ and the angle of incidence $\alpha$. In Fig. 5c and Fig. 5d the error is plotted for the TE and TM cases versus the electrical curvature of the cylinder $\kappa / k_{0}$ and the electrical torsion $\tau / k_{0}$.

It is seen that the approximation is precise for large structures at low angles of incidence. A good rule of thumb for the validity could be that $k_{0} a>\pi$ or equivalently $a>\lambda_{0} / 2$ and $\alpha<60^{\circ}$. If this is converted to general geometric parameters it corresponds to $\frac{\kappa}{\kappa^{2}+\tau^{2}}>\lambda_{0} / 2$ and $\tau / \kappa<2$. The inaccuracy in the paraxial region might be caused by modes, that are not captured by this approximation. The fields in the paraxial region for an IBC cylinder is discussed in [32]. Following the approach outlined in [32] it might be possible to derive accurate results in the paraxial region for a lossy dielectric cylinder as well.

To further investigate the validity of the proposed approximation, the relative root-mean-square error for a sweep of the imaginary part of $-k_{t 1} a$ and the radius of 
the cylinder $a$ has been calculated. For the approximation in Eq. 17 to be valid the imaginary part of $k_{t 1} a$ has to be large which is equivalent to the cylinder being opaque. The cylinder's permittivity was kept constant at $\epsilon_{r}=39.2$, the solution frequency was $2.45 \mathrm{GHz}$, and the angle of incidence was kept at $\alpha=0^{\circ}$. If these parameters are changed the result is only a little different, but the conclusion remains the same. The decibel value of the RMS-error is shown in Fig. 6.

The proposed approximation is seen to be valid as long as the imaginary part of $-k_{t 1} a$ is large. For smaller cylinders and for TE incident waves the approximations remains valid for even a small imaginary part of around 2. A possible explanation of the improved area of validity for small cylinders could be the fact that the path through the cylinder is a combination of the reflection on the interfaces and the attenuation through the cylinder. For small cylinders the path through the cylinder is weaker compared to the path around than it is for larger cylinders caused by the initial high loss due to the reflections. The lower loss in the TE creeping waves is believed to be the cause of the improved area of validity for the case of a TE incident wave compared to the case of a TM incident wave. It is seen that for small $k_{0} a$ the approximation breaks down as quantified from Fig. 5.

\section{GTD Formulation FOR GENERAL GeOMETRY}

The expressions for the diffracted electric and magnetic fields on and away from the cylinder can be described by the use of a few constants. The coupling between the TE and TM mode in air and the modes on the cylinder are given by the square root of $D_{p}^{\mathrm{e}}$ and $D_{p}^{\mathrm{m}}$. This can be realized by using the first order Debye expansion of the Hankel functions in Eq 19. Thereby the diffracted field $\vec{E}^{\text {d }}$ in the deep shadow region away from the cylinder, caused by an incident plane wave $\vec{E}^{\mathrm{i}}$, can be described as:

$$
\vec{E}^{\mathrm{d}} \sim \vec{E}^{\mathrm{i}} \cdot \overline{\bar{T}} \frac{e^{-j k_{0} s}}{\sqrt{s}} e^{-j k_{0} t-\alpha_{p} t}
$$

where

$$
\overline{\bar{T}} \sim \sum_{p=1}^{\infty}\left(\hat{b}^{\prime} \hat{b} D_{p}^{\mathrm{m}}+\hat{b}^{\prime} \hat{n} D_{p}^{\mathrm{c}}-\hat{n}^{\prime} \hat{b} D_{p}^{\mathrm{c}}+\hat{n}^{\prime} \hat{n} D_{p}^{\mathrm{e}}\right)
$$

where $\left(\hat{t}^{\prime}, \hat{n}^{\prime}, \hat{b}^{\prime}\right)$ are the tangential, normal, and binormal unit vectors, respectively, at the source or ingoing grazing point and similarly $(\hat{t}, \hat{n}, \hat{b})$ are the unit vectors at the observation or outgoing grazing point. The shift in sign of $D_{p}^{\mathrm{c}}$ is explained by the shift of direction of $\hat{b}$ dependent on the way of travel. The equation can be rewritten to:

$$
\overline{\bar{T}} \sim \sum_{p=1}^{\infty}\left(\hat{b}^{\prime} D_{p, \mathrm{i}}^{\mathrm{m}}+\hat{n}^{\prime} D_{p, \mathrm{i}}^{\mathrm{e}}\right)\left(\hat{b} D_{p, \mathrm{o}}^{\mathrm{m}}+\hat{n} D_{p, \mathrm{o}}^{\mathrm{e}}\right)
$$

where the 'i' and 'o' subscript refers to incoming or outgoing waves with respect to the cylinder. The magnitudes of the constants are given by:

$$
\begin{aligned}
& \left|D_{p, \mathrm{i}}^{\mathrm{m}}\right|=\left|D_{p, \mathrm{o}}^{\mathrm{m}}\right|=\left|\sqrt{D_{p}^{\mathrm{m}}}\right| \\
& \left|D_{p, \mathrm{i}}^{\mathrm{e}}\right|=\left|D_{p, \mathrm{o}}^{\mathrm{e}}\right|=\left|\sqrt{D_{p}^{\mathrm{e}}}\right|
\end{aligned}
$$

where phases are chosen in such a way that:

$$
\begin{aligned}
& D_{p, \mathrm{i}}^{\mathrm{m}} D_{p, \mathrm{o}}^{\mathrm{m}}=D_{p}^{\mathrm{m}} \\
& D_{p, \mathrm{i}}^{\mathrm{m}} D_{p, \mathrm{o}}^{\mathrm{e}}=D_{p}^{\mathrm{c}} \\
& D_{p, \mathrm{i}}^{\mathrm{e}} D_{p, \mathrm{o}}^{\mathrm{m}}=-D_{p}^{\mathrm{c}} \\
& D_{p, \mathrm{o}}^{\mathrm{e}} D_{p, \mathrm{i}}^{\mathrm{e}}=D_{p}^{\mathrm{e}}
\end{aligned}
$$

The fields on the cylinder from an incident plane wave can be described as well. By evaluating Eq. 19 for points on the surface the so-called attachment coefficients can be determined. In the deep shadow on the cylinder the diffracted fields $\vec{E}^{\mathrm{d}}$ from an incident plane wave $\vec{E}^{\mathrm{i}}$ will be given by:

$$
\vec{E}^{\mathrm{d}} \sim \vec{E}^{\mathrm{i}} \cdot \overline{\bar{S}} e^{-j k_{0} t-\alpha_{p} t}
$$

where

$$
\overline{\bar{S}} \sim \sum_{p=1}^{\infty}\left(\hat{b}^{\prime} D_{p, \mathrm{i}}^{\mathrm{m}}+\hat{n}^{\prime} D_{p, \mathrm{i}}^{\mathrm{e}}\right)\left(\hat{t} A_{p, \mathrm{t}}^{\mathrm{e}}+\hat{b} A_{p, \mathrm{~b}}^{\mathrm{e}}+\hat{n} A_{p, \mathrm{n}}^{\mathrm{e}}\right)
$$

with the attachment coefficient given by (The argument, $k_{\mathrm{t} 0} a$, of the Hankel functions has been suppressed):

$$
\begin{aligned}
A_{p, \mathrm{n}}^{\mathrm{e}}= & \sqrt{\frac{k_{0} \pi}{2 j}} \cos \alpha\left(\frac{v}{k_{\mathrm{t} 0} a} D_{p, \mathrm{o}}^{\mathrm{e}} H_{v}^{(2)}-j \sin \alpha D_{p, \mathrm{o}}^{\mathrm{m}} H_{v}^{(2) \prime}\right) \\
A_{p, \mathrm{~b}}^{\mathrm{e}}= & \sqrt{\frac{k_{0} \pi}{2 j} \cos \alpha\left[j \sin \alpha D_{p, \mathrm{o}}^{\mathrm{e}} H_{v}^{(2) \prime}\right.} \\
& \left.+\left(1+\frac{m_{\mathrm{t}} \tau_{p} \sin ^{2} \alpha}{k_{\mathrm{t} 0} a}\right) D_{p, \mathrm{o}}^{\mathrm{m}} H_{v}^{(2)}\right] \\
A_{p, \mathrm{t}}^{\mathrm{e}}= & \sqrt{\frac{k_{0} \pi}{2 j} \cos \alpha\left[-j \cos \alpha D_{p, \mathrm{o}}^{\mathrm{e}} H_{v}^{(2) \prime}\right.} \\
& \left.-\left(\frac{m_{\mathrm{t}} \tau_{p} \cos \alpha \sin \alpha}{k_{\mathrm{t} 0} a}\right) D_{p, \mathrm{o}}^{\mathrm{m}} H_{v}^{(2)}\right]
\end{aligned}
$$

Equivalently the diffracted fields $\vec{E}^{\text {d }}$ in the shadow part of the far-field from a point source $\vec{J}_{\mathrm{i}}$ on the surface can be found by reciprocity to be:

$$
\vec{E}^{\mathrm{d}} \sim \frac{j k_{0} \eta_{0}}{4 \pi} \vec{J}_{\mathrm{i}} \cdot \overline{\bar{U}} \frac{e^{-j k_{0} t-\alpha_{p} t}}{\sqrt{t+s}} \frac{e^{-j k_{0} s}}{\sqrt{s}}
$$

where

$$
\overline{\bar{U}} \sim \sum_{p=1}^{\infty}\left(\hat{t}^{\prime} L_{p, \mathrm{t}}^{\mathrm{e}}+\hat{b}^{\prime} L_{p, \mathrm{~b}}^{\mathrm{e}}+\hat{n}^{\prime} L_{p, \mathrm{n}}^{\mathrm{e}}\right)\left(\hat{b} D_{p, \mathrm{o}}^{\mathrm{m}}+\hat{n} D_{p, \mathrm{o}}^{\mathrm{e}}\right)
$$




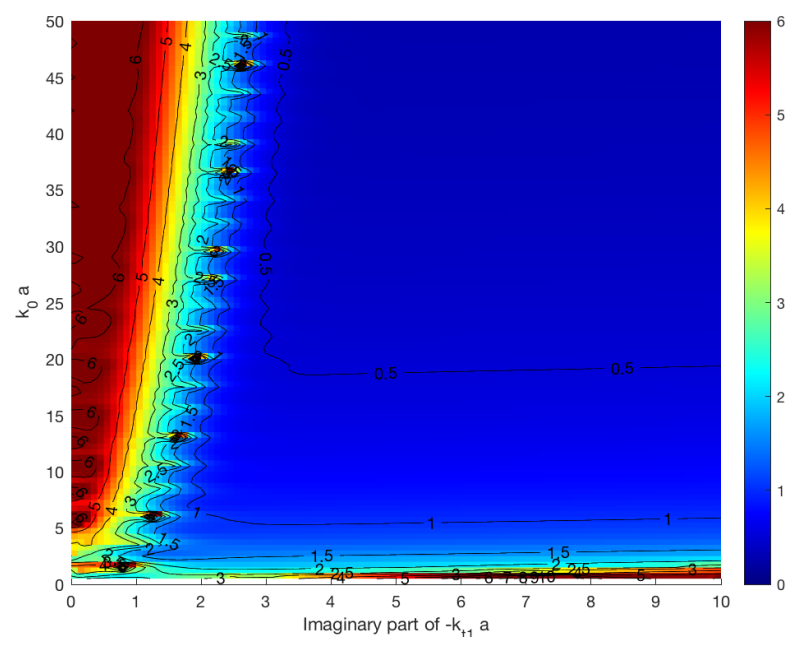

(a)

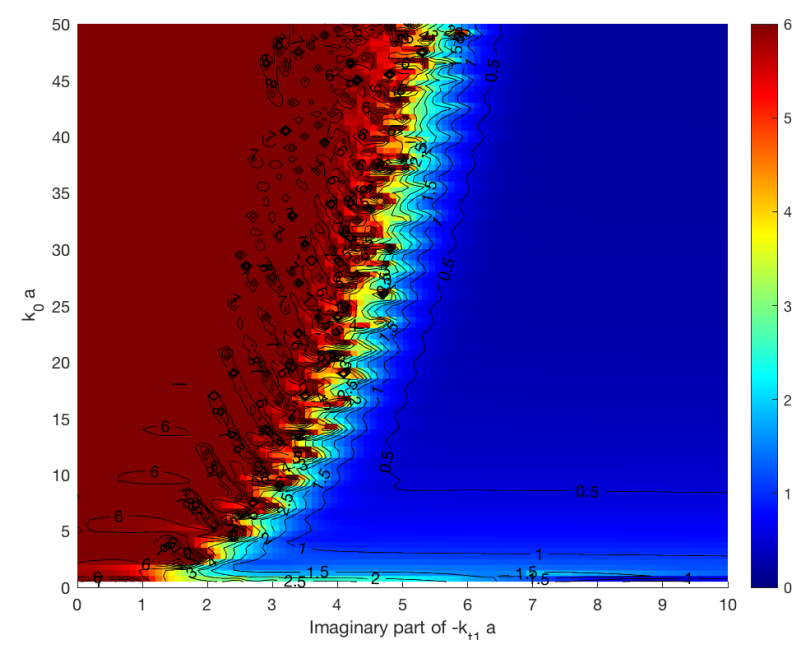

(b)

Fig. 6. Root-mean-square of the relative error (from zero (blue) to six (red) decibel) in the electric field in the deep shadow for different cylinders. As a function of the imaginary part of the $k_{t 1} a$ and electrical length of the cylinder $k_{0} a$ in the TE (a) and TM (b) cases.

The launching coefficients are given by:

$$
\begin{aligned}
L_{p, \mathrm{n}}^{\mathrm{e}}= & \sqrt{\frac{k_{0} \pi}{2 j}} \cos \alpha\left(\frac{v}{k_{\mathrm{t} 0} a} D_{p, \mathrm{i}}^{\mathrm{e}} H_{v}^{(2)}+j \sin \alpha D_{p, \mathrm{i}}^{\mathrm{m}} H_{v}^{(2) \prime}\right) \\
L_{p, \mathrm{~b}}^{\mathrm{e}}= & \sqrt{\frac{k_{0} \pi}{2 j} \cos \alpha\left[j \sin \alpha D_{p, \mathrm{i}}^{\mathrm{e}} H_{v}^{(2) \prime}\right.} \\
& \left.-\left(1+\frac{m_{\mathrm{t}} \tau_{p} \sin ^{2} \alpha}{k_{\mathrm{t} 0} a}\right) D_{p, \mathrm{i}}^{\mathrm{m}} H_{v}^{(2)}\right] \\
L_{p, \mathrm{t}}^{\mathrm{e}}= & \sqrt{\frac{k_{0} \pi}{2 j} \cos \alpha\left[-j \cos \alpha D_{p, \mathrm{i}}^{\mathrm{e}} H_{v}^{(2) \prime}\right.} \\
& \left.+\left(\frac{m_{\mathrm{t}} \tau_{p} \cos \alpha \sin \alpha}{k_{\mathrm{t} 0} a}\right) D_{p, \mathrm{i}}^{\mathrm{m}} H_{v}^{(2)}\right]
\end{aligned}
$$

By the use of the launching and attachment coefficients the field on the cylinder caused by a source $\vec{J}_{\mathrm{i}}$ on the cylinder can be found as:

$$
\vec{E}^{\mathrm{d}} \sim \frac{j k_{0} \eta_{0}}{4 \pi} \vec{J}_{\mathrm{i}} \cdot \overline{\bar{V}} \frac{e^{-j k_{0} t-\alpha_{p} t}}{\sqrt{t}}
$$

where

$$
\overline{\bar{V}} \sim \sum_{p=1}^{\infty}\left(\hat{t}^{\prime} L_{p, \mathrm{t}}^{\mathrm{e}}+\hat{b}^{\prime} L_{p, \mathrm{~b}}^{\mathrm{e}}+\hat{n}^{\prime} L_{p, \mathrm{n}}^{\mathrm{e}}\right)\left(\hat{t} A_{p, \mathrm{t}}^{\mathrm{e}}+\hat{b} A_{p, \mathrm{~b}}^{\mathrm{e}}+\hat{n} A_{p, \mathrm{n}}^{\mathrm{e}}\right)
$$

The coefficients first determined from the plane wave incidence case matches with the ones from the infinitesimal dipole source case. This can be seen by comparing to Eq. 26 .

The behavior of the creeping wave on the body has been described by the use of the unit vectors, which are defined by the geodesic paths. The only thing that remains is to replace $a$, $\cos \alpha$ and $\sin \alpha$ with expressions not specific to the cylinder.
This can be done by the use of:

$$
\begin{aligned}
a & =\frac{\kappa}{\kappa^{2}+\tau^{2}} \\
\cos \alpha & =\frac{\kappa}{\sqrt{\kappa^{2}+\tau^{2}}} \\
\sin \alpha & =\frac{-\tau}{\sqrt{\kappa^{2}+\tau^{2}}}
\end{aligned}
$$

where $\kappa$ and $\tau$ is the surface ray curvature and torsion, respectively. Furthermore, when the term $\frac{1}{\sqrt{t}}$ or $\frac{1}{\sqrt{t+s}}$ appears one should replace it with:

$$
\frac{1}{\sqrt{t}} \sqrt{\frac{d \chi_{0}}{d \chi}}=\sqrt{\frac{d \psi_{0}}{d \chi}}
$$

where $d \chi$ and $d \chi_{0}$ are the distance between adjacent rays at the observation and source point, respectively. $d \psi_{0}=d \chi_{0} / t$ is the angle between adjacent rays at the source. Finally, the attenuation will be variable over the surface since the curvature and torsion along a geodesic may change for a general geometry contrary to a cylinder. Therefore, the term $\alpha_{p} t$ should be replaced by $\int_{0}^{t} \alpha_{p}\left(t^{\prime}\right) \mathrm{d} t^{\prime}$. From these equations the fields in the proximity of the human body or any opaque lossy dielectric object and in the deep shadow can be determined for any convex geometry.

\section{CONCLUSION}

A general geometrical theory of diffraction formulation of the on-body propagation was found. The canonical problem of an infinitely long cylinder was solved. It was done for plane wave incidence as well as for a magnetic or an electric infinitesimal dipole source on the surface of the cylinder. The exact solution was transformed to an asymptotic form valid in the deep shadow region of an opaque electrically large human body or lossy dielectric cylinder with radius $a$. The model was shown to be valid as long as $a>\lambda_{0} / 2$ and $\alpha<60^{\circ}$ or $\frac{\kappa}{\kappa^{2}+\tau^{2}}>\lambda_{0} / 2$ and $\tau / \kappa<2$. Furthermore, the improvement by the use of this model compared to a PEC 
approximation of the human body was significant. Especially, for the TM case and torsional cases. The improvement over an IBC model was smaller, but significant for low dielectric constant tissue such as fat. The asymptotic formulation for the cylinder was generalized to any convex geometry. It was shown that the two different excitations of the cylinder resulted in the same generalized constants. Future work could be to solve other canonical geometries to further validate the model. The relevant cases would especially be an ellipsoid and an elliptical cylinder. A layered model that might describe the human body more accurately could be relevant future work as well.

\section{REFERENCES}

[1] R. Chandra and A. J. Johansson, "A link loss model for the on-body propagation channel for binaural hearing aids," IEEE Trans. Antennas Propagat., vol. 61, no. 12, pp. 6180-6190, 2013.

[2] E. Plouhinec, B. Uguen, M. Mhedhbi, and S. Avrillon, "3D UTD modeling of a measured antenna disturbed by a dielectric circular cylinder in WBAN context," in 2014 IEEE 79th Vehicular Technology Conference (VTC Spring), May 2014, pp. 1-5.

[3] S. H. Kvist, J. Thaysen, and K. B. Jakobsen, "Ear-to-ear on-body channel model for hearing aid applications," IEEE Transactions on Antennas and Propagation, vol. 63, no. 1, pp. 344-352, 2015.

[4] Y. Zhao, Y. Hao, A. Alomainy, and C. G. Parini, "UWB On-Body Radio Channel Modeling Using Ray Theory and Subband FDTD Method," IEEE Trans. Microwave Theory Tech., vol. 54, no. 4, pp. 1827-1835, Apr 2006

[5] R. Chandra and A. J. Johansson, "An Analytical Link-Loss Model for On-Body Propagation Around the Body Based on Elliptical Approximation of the Torso With Arms' Influence Included," IEEE Antennas Wirel. Propag. Lett., vol. 12, pp. 528-531, Apr 2013.

[6] M. Ghaddar, L. Talbi, and T. A. Denidni, "Human Body Modelling for Prediction of Effect of People on Indoor Propagation Channel," Electronics Letters, vol. 40, no. 25, pp. 1592-1594, Dec 2004.

[7] T. E. P. Alves, B. Poussot, and J.-M. Laheurte, "Analytical Propagation Modeling of BAN Channels Based on the Creeping-Wave Theory," IEEE Trans. Antennas Propagat., vol. 59, no. 4, pp. 1269-1274, Apr 2011.

[8] C. Oliveira and L. M. Correia, "A Statistical Model to Characterize User Influence in Body Area Networks," in Vehicular Technology Conference Fall (VTC 2010-Fall), 2010 IEEE 72nd, Sep 2010.

[9] N. P. B. Kammersgaard, S. H. Kvist, J. Thaysen, and K. B. Jakobsen, "Validity of PEC approximation for on-body propagation," 2016 10th European Conference on Antennas and Propagation (EuCAP), 2016.

[10] _ - "Electromagnetic fields at the surface of human-body cylinders," Proceedings of the 2016 International Workshop on Antenna Technology, pp. 170-173, 2016.

[11] A. G. Aguilar, P. H. Pathak, and M. Sierra-Perez, "A canonical UTD solution for electromagnetic scattering by an electrically large impedance circular cylinder illuminated by an obliquely incident plane wave," IEEE Transactions on Antennas and Propagation, vol. 61, no. 10, pp. 51445154, 2013.

[12] A. G. Aguilar, Z. Sipus, and M. Sierra-Perez, "An asymptotic solution for surface fields on a dielectric-coated circular cylinder with an effective impedance boundary condition," IEEE Transactions on Antennas and Propagation, vol. 61, no. 10, pp. 5175-5183, 2013.

[13] K. Naishadham and L. B. Felsen, "Dispersion of waves guided along a cylindrical substrate superstrate layered medium," IEEE Transactions on Antennas and Propagation, vol. 41, no. 3, pp. 304-313, 1993.

[14] K. Naishadham and J. E. Piou, "Analytical characterization and validation of creeping waves on dielectric coated and perfectly conducting cylinders," Radio Science, vol. 45, no. 5, 2010.

[15] M. Bosiljevac, Z. Sipus, and A. K. Skrivervik, "Propagation in finite lossy media: An application to WBAN," IEEE Antennas and Wireless Propagation Letters, vol. 14, pp. 1546-1549, 2015.

[16] L. B. Felsen and N. Marcuvitz, Radiation and scattering of waves. Prentice-Hall, 1973.

[17] S. Gabriel, R. W. Lau, and C. Gabriel, "The Dielectric Properties of Biological Tissues: III. Parametric Models for the Dielectric Spectrum of Tissues," Physics in Medicine and Biology, Nov 1996.
[18] "IEEE recommended practice for determining the peak spatial-average specific absorption rate (SAR) in the human head from wireless communications devices: Measurement techniques," 2013.

[19] B. R. Levy and J. B. Keller, "Diffraction by a Smooth Object," Communications on Pure and Applied Mathematics, vol. 12, no. 1, pp. 159-209, 1959.

[20] J. R. Wait, "Scattering of a plane wave from a circular dielectric cylinder at oblique incidence," Canadian Journal of Physics, vol. 33, no. 5, pp. 189-195, 1955.

[21] R. F. Harrington, Time-Harmonic Electromagnetic Fields. John Wiley \& Sons, 2001.

[22] C. Tokgoz and R. J. Marhefka, "A UTD based asymptotic solution for the surface magnetic field on a source excited circular cylinder with an impedance boundary condition," IEEE Transactions on Antennas and Propagation, vol. 54, no. 6, pp. 1750-1757, 2006

[23] B. Alisan, V. B. Erturk, and A. Altintas, "Efficient computation of nonparaxial surface fields excited on an electrically large circular cylinder with an impedance boundary condition," IEEE Transactions on Antennas and Propagation, vol. 54, no. 9, pp. 2559-2567, 2006.

[24] P. H. Pathak and R. G. Kouyoumjian, "An analysis of the radiation from apertures in curved surfaces by the geometrical theory of diffraction," Proceedings of the IEEE, vol. 62, no. 11, pp. 1438-47, 1438-1447, 1974.

[25] P. H. Pathak, N. Wang, W. D. Burnside, and R. G. Kouyoumjian, "A uniform gtd solution for the radiation from sources on a convex surface," IEEE Transactions on Antennas and Propagation, vol. AP-29, no. 4, pp. 609-22, 609-622, 1981

[26] P. H. Pathak and N. Wang, "Ray analysis of mutual coupling between antennas on a convex surface," IEEE Transactions on Antennas and Propagation, vol. 29, no. 6, pp. 911-922, 1981.

[27] A. Fort, F. Keshmiri, G. R. Crusats, C. Craeye, and C. Oestges, "A body area propagation model derived from fundamental principles: Analytical analysis and comparison with measurements," IEEE Transactions on Antennas and Propagation, vol. 58, no. 2, pp. 503-514, 2010

[28] R. Paknys, "Evaluation of hankel functions with complex argument and complex-order," IEEE Transactions on Antennas and Propagation, vol. 40 , no. 5 , pp. $569-578,1992$.

[29] G. Watson, "The diffraction of electric waves by the earth," Proceedings of the Royal Society of London Series A-containing Papers of a Mathematical and Physical Character, vol. 95, no. 666, pp. 83-99, 1918.

[30] V. Fock, "Diffraction of radio waves around the earths surface," Zhurnal Eksperimentalnoi I Teoreticheskoi Fiziki, vol. 15, no. 9, pp. 479-496, 1945.

[31] P. H. Pathak and N. Wang, "An analysis of the mutual coupling between antennas on a smooth convex surface," Final Report 784583-7, pp. The Ohio State University ElectroScience Lab., Dep. Elec. Eng., 1978.

[32] C. Tokgoz, P. Pathak, and R. Marhefka, "An asymptotic solution for the surface magnetic field within the paraxial region of a circular cylinder with an impedance boundary condition," IEEE Transactions on Antennas and Propagation, vol. 53, no. 4, pp. 1435-1443, 2005.

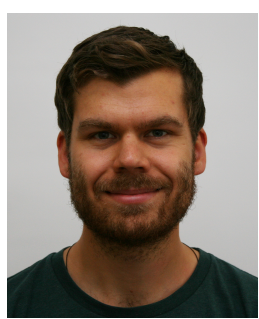

Nikolaj Peter Brunvoll Kammersgaard received the B.Sc. and M.Sc. degrees in electrical engineering from the Technical University of Denmark, Lyngby, in 2013 and 2014, respectively. Since 2014, he has been affiliated with GN Hearing A/S, a Danish hearing aid manufacturer, where he is currently pursuing an Industrial Ph.D. degree in cooperation with the Technical University of Denmark. His research interests include on-body antennas and propagation, as well as electrically small antennas and physical limitations of antennas. He was awarded the Student Paper Award for best student paper at the International Workshop on Antenna Technology 2015. In 2015, he was awarded with "Kandidatprisen" from the Danish Association of Engineers (IDA). The award is given to three recently graduated promising electrical engineers for their Master's thesis. 


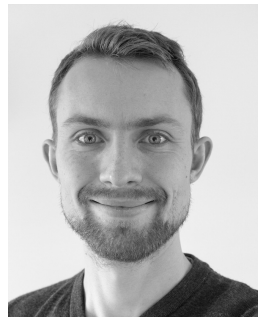

Søren Helstrup Kvist received the B.Sc., M.Sc. and Ph.D. degrees in electrical engineering from the Technical University of Denmark in 2007, 2009 and 2014, respectively. Since 2009 he has been affiliated with GN Hearing A/S, a Danish hearing aid manufacturer, where he is currently employed as manager of the Radio Systems group. His research interests include on-body antennas and propagation, as well as electrically small antennas and physical limitations of antennas. Dr. Kvist was awarded the "Elektroprisen 2015" from the Danish Association of Engineers (IDA). The award is given for excellent technical and scientific work within electrical engineering.

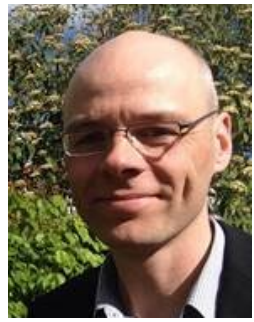

Jesper Thaysen received the B.Sc., M.Sc. and Ph.D. degrees in electrical engineering from the Technical University of Denmark in 1998, 2000 and 2005, respectively, and an MBA from Middlesex University in 2015. Since 2008 he has been employed at GN Hearing A/S, a Danish hearing aid manufacturer, where he currently acts as the Head of the Global Manufacturing Organization. His research interests include small antennas and on-body antennas and propagation. Dr. Thaysen has overseen more than 40 B.Sc. and M.Sc. students, as well as $3 \mathrm{Ph} . \mathrm{D}$. students, as the company representative in university-industry cooperative projects.

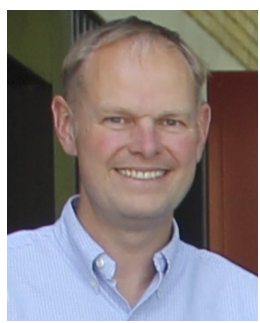

Kaj Bjarne Jakobsen received the B.Sc.EE and the M.Sc.EE degree from the Technical University of Denmark, Kgs. Lyngby, in 1985 and 1986, respectively, the Ph.D. degree in Electrical Engineering from University of Dayton, Dayton, $\mathrm{OH}$, in 1989, and the HD in Organization and Management, Copenhagen Business School, Copenhagen in 2000. From 1986-1989 he was a Fulbright Scholar at the Department of Electrical Engineering, University of Dayton, OH. Since 1990 he has been with the Department of Electrical Engineering, Technical University of Denmark, Kgs. Lyngby, where he is Associate Professor. His research interests are in body-centric wireless network, wireless body area network, and body sensor network. He received in 1989 the NCR Stakeholder Award, Ohio, USA, and was appointed Teacher-of-the-Year at the Technical University of Denmark in 1994. 